\title{
Physical Exhaustion Induced Variations in Event-Related Potentials and Cognitive Task Performance in Young Adults
}

\author{
Afreen Begum H. Itagi ${ }^{a}$ Navin A. Patil ${ }^{b}$ Rahul K. Kotian ${ }^{c}$ Suneel Kumar Reddy \\ Shardul Abhyankare Reena Sherin Parveen ${ }^{b}$ \\ ${ }^{a}$ Department of Physiology, All India Institute of Medical Sciences, Mangalagiri, India; ${ }^{b}$ Department of \\ Pharmacology, K.M.C. Manipal, Manipal University, Manipal, India; ' ${ }^{C}$ epartment of Medical Imaging Technology, \\ Manipal School of Allied Health Sciences, Manipal University, Manipal, India; ${ }^{d}$ Department of Pharmacology,

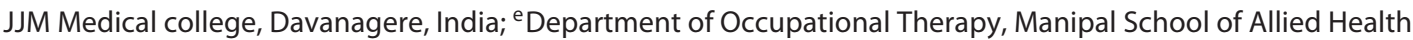 \\ Sciences, Manipal University, Manipal, India
}

\section{Keywords}

Event related potential - Cognitive performance .

Exhaustion · Stroop test

\begin{abstract}
Background/Aims: Physical exhaustion is not always peripheral, and it is the brain that causes the sensation of fatigue either due to decrease of metabolic resources or due to central activation process that regulates attention and performance. This study was undertaken to observe the variations in event-related potentials (ERPs) and cognitive performance after an exhausting physical exercise. Methods: A total of 60 healthy young adult subjects were included in the study. The study was conducted in 2 phases with at least a week gap between the phases. The participants answered a Multidimensional Fatigue Inventory (MFI-20) questionnaire before and after trials in each phase to measure the induced physical exhaustion. In phase I (control trial), the ERP data were processed using P300, Standard auditory "oddball paradigm," on computerized evoked potential recorder (RMS EMG MK-2) using 10/20 system to know the engagement of
\end{abstract}

attention after which participants were given to perform cognitive tasks such as "Stroop Test, Trial Making Test and Mini Mental State Examination." In Phase II (exercise trial), the participants were instructed to cycle as hard as they could, till they could not continue anymore, which was followed by recording of P300-evoked potentials and performance of cognitive tasks as in Phase I. Paired $t$ test was used to compare between any dependent variables. Results: Fatigue-related subjective measures (MFI-20) showed that both mental and physical exhaustion were significantly greater in the exercise-involved cognitive trial than in the control trial. Lower P300 latencies reflect faster reaction time; however, their response accuracies were poorer resulting in poorer cognitive performances. Participants subjected to control trial performed better in terms of higher percentage accuracy but with slow reaction time. Conclusion: The participants experienced more fatigue physically and mentally during the exercise that involved cognitive tasks. An apparent decrease in attention based on decreased percentage accuracy of response was evident, implying that fatigue, performance, and attention are interdependent.

(c) 2019 S. Karger AG, Base

Dr. Afreen Begum H. Itag

Department of Physiology

All India Institute of Medical Sciences

Mangalagiri (India)

E-Mail afreen.itagi@gmail.com 


\section{Introduction}

Everything about our existence in today's world is moving at such a fast pace that it just leaves many people in our modern society feeling overwhelmingly exhausted $[1,2]$. Exhaustion is defined as a decline in the ability and efficiency of mental and/or physical activities that are caused by excessive mental and/or physical activities. Fatigue can be either physical or mental and is often accompanied by peculiar sense of discomfort, desire to rest, and reduced motivation. Fatigue can manifest in potentially impaired cognitive function and has been shown to involve more complex neural mechanisms related to cognitive task performance $[1,3]$.

The epidemiology of chronic fatigue has mainly been described in developed and developing countries, where a considerable proportion of people are often found to have fatigue-related somatoform disorders or mental disorders such as depression and anxiety $[4,5]$. Previous research on specific group of people (pilots, drivers, and so on), engaged in work requiring a good attention and accuracy, has reported difficulties with concentration and attention during tasking when they are fatigued, resulting in adverse consequences on task performance $[2,6-8]$.

Attention, a prominent feature of dynamic human behavior, is particularly affected by mental exhaustion. Lack of attention results in improper (biased) processing of incoming information for its relevance [9]. Event-related potential (ERP) measures (P300) are directly related to the allocation of attention resources during stimulus engagement with increased latency indicating longer processing time [10]. Available meta-analysis and review associates increased amplitude and short latency relative to basal rate following bouts of exercise in adults, indicating significant improvement in cognition $[11,12]$.

To date there have been few research on the effect of exhaustion on cognition throughout the world. Comparatively little is known about the phenomenon and physiology of cognitive fatigue. However, the existing literature has demonstrated that specific task and intensity of task induce fatigue, which in turn influences the performance [13-15]. Further neurophysiological studies support a possible role of fatigue in mediating many of the behavioral effects associated with cognitive performance $[16,17]$.

The effect of physical activity on cognition has grown in interest in recent years with substantial reports indicating a beneficial effect on cognitive processes through isolated acute bouts of exercise $[18,19]$. The physical activity has also been observed to bring an increase in cogni- tive performance. However, the conflicting studies do exist reporting limited or no influence of physical activity on cognition $[20,21]$.

In many respects, it appears that the effect of physical exhaustion on attention and performance is still inconsistent and not documented in Indian population. Hence, the present study is undertaken as an attempt to explore the effect of an exhausting physical exercise on cognitive performance among young adults.

\section{Methodology}

This cross-sectional interventional study was conducted in Davangere, India. The selected subjects were apparently healthy young adults aged $21.5-31.9$ years. A thorough history about existing medical conditions and medications taken in past 6 months was obtained followed by clinical and systemic examinations. Subjects with established cardiopulmonary diseases, unstable coronary syndromes, and on vigorous athletic training exercises were excluded.

The sample required was estimated using the formula $n=$ $\left(Z_{a / 2}\right)^{2} s^{2} / d^{2}$, the pilot study conducted on 5 healthy young adults, yielded a SD of 4.7 at confidence interval of $95 \%$ and error rate of $5 \%$ resulted in the minimum sample size required to be 27 subjects; however, after allowance of additional $10 \%$, the corrected final sample size was estimated to be 30 subjects. However, a total of 60 (30 male and 30 female) participants were included in the study after obtaining written informed consent in accordance with the Institutional Review Board approved protocol after being explained the purpose and procedures in detail.

\section{Multidimensional Fatigue Inventory-20}

The Multidimensional Fatigue Inventory (MFI) was used for the assessment of trait exhaustion or fatigue: general fatigue, physical fatigue, reduced activity, reduced motivation, and mental fatigue $[22,23]$. The reliability of the MFI-20 questionnaire items was tested using Cronbach's alpha, which was found to be 0.82 indicating MFI as a reliable tool for assessing the subjective fatigue. MFI-20 questionnaire was administered to be answered by the participants before and after the cognitive task performance in both phases to assess the different aspects of exhaustion and to compare any changes in the subjective feeling measures between pre and post the cognitive test (tasks).

\section{P300 Evoked Potential}

The P300 is an evoked potential associated to engagement of attention and it is linked to an individual's reaction time to an external stimulus a well as it can be used to measure how demanding a task is on the cognitive workload [24, 25]. In this study ERP data were processed using P300 Standard auditory "oddball paradigm" on fully computerized-evoked potential recorder (RMS EMG MK-2; RMS RECORDERS and MEDICARE SYSTEM Chandigarh) using 10/20 system to know the engagement of attention. Test was carried out in pre-cooled, quiet, dimly lit room with sitting comfortably facing the computer monitor. The acoustically shielded THD 32 ear phones were placed on the 
ear and head bands were adjusted. Monoaural auditory stimulus consisting of rare faction clicks of 100 microseconds with intensities starting from 30 to $100 \mathrm{~dB}$ were delivered through electrically shielded earphones at a rate of $11.1 / \mathrm{s}$. Contra lateral ear was masked. Throughout trial, the participant has to focus attention on one of the 36 characters of the matrix. The random sequence of 6 row and 6 column flashes constitutes an oddball paradigm, with the row and the column containing the desired character constituting the rare set. The thorough description is similar to as described elsewhere [10]. The reaction time measurements were performed when the subject was seated in front of the device and asked to press the button as soon as the light appeared. The RT was measured as the interval of time $(\mathrm{ms})$ between the appearance of the light and pressing the button. The mean value of 5 repetitions was calculated each time and was taken for the analysis.

\section{Description of the Cognitive Tasks}

The Stroop test, trial making test (TMT), and Mini Mental State Examination (MMSE) were used as cognitive tasks. These tests place psychological strain of mainly cognitive nature, thereby denuding a sustained attention from participants during entire tasks for them to be accurate and quick in their responses.

A computerized cued Stroop task was performed [26]. In this task, an instructional cue is presented on each trial ("word" or "color") and followed by a 1, 3, or 5 s cue-target interval and an imperative color word stimulus ("red," "blue," or "green") written in colored letters. For the "word," the task participants were instructed to read the word, whereas in the "color," task participants were instructed to name the color of the letters. In total, $60 \%$ of the trials were congruent (e.g., "red" written in red letters) and 40\% were incongruent (e.g., "red" written in green letters).

MMSE was used in quantitatively estimating the severity of cognitive impairment, in serially documenting cognitive change. MMSE was scored as indicated by Folstein et al. [27]. The score is the total number of correct answers where a score of 23 or less generally has been accepted as indicating the presence of cognitive impairment [28].

TMT [29] (or divided attention test) was used to evaluate information processing speed, visual scanning ability, and other cognitive processing and executive functioning performed in 2 parts ( $A$ and $B$ ). In part $A$, the participant is given a white sheet of paper with 2 circles on it, numbered 1 through 25 , and asked to connect the circles in numerical sequence as quickly as possible. In part B, there are again 25 circles, numbered from 1 through 13 and lettered from A to L, the subject is required to connect the circles, again in sequences, but alternating the letters alphabetically in between. The scores represent the number of seconds required to finish each part.

\section{Procedure}

The selected participants were invited at different timings so as to reduce any learning effect (Lord et al. 1998) [30]. The study was conducted in 2 phases with at least a week gap between the phases.

\section{Phase-I (Control Phase)}

In this first phase, participants initially completed the MFI-20 questionnaire followed by processing the ERP data using P300 and the performance of cognitive tasks, namely, "Stroop Test, TMT, and MMSE", after which lastly again MFI-20 was administered after the completion of cognition tasks.

\section{Phase-II (Exercise Phase)}

In this phase, after initially answering the MFI-20 questionnaire, the participants were instructed to cycle as hard as they could, till they could not continue anymore, which was followed by recording of P300-evoked potential and performance of the cognitive tasks and as in the control phase. Finally after completing the cognitive tasks, they again filled the MFI-20 questionnaire.

\section{Statistical Analysis}

The results are expressed as mean \pm SD or percentages. SPSS software version 19.0 (SPSS Inc., IBM Corporation, Chicago, IL, USA) was used for analysis. Paired $t$ test was used to compare between any dependent variables for significant differences. $p<0.05$ was considered significant.

\section{Results}

The results are described in separate sections namely the subjective measure consisting of the MFI-20 assessing the general fatigue, physical fatigue, mental fatigue, reduced motivation, reduced activity; ERP analysis results followed by the cognitive performance of the participants in terms of both reaction time and percentage accuracy of responses.

\section{MFI-20 Subjective Measures}

Fatigue-related subjective measures (MFI-20) showed that both mental and physical exhaustion were significantly greater in the exercise-phase cognitive trial than in the control phase. Table 1 observes the summarized results of the each subjective measures before and after the performance of cognitive tasks, and a significant difference was observed between the means for post control and post exercise experimental condition $(p<0.05)$.

\section{P300 Event-Related Potentials and Cognitive \\ Performance}

Lower P300 latencies reflect faster reaction time, but their response accuracies were poorer resulting in poorer cognitive performances (Fig. 1,2).

The mean reaction time (Table 2 ) of the participants in responding to the visual cues during the cognitive task for the control phase and exercise phase was $470 \pm 19.1$ and $405.2 \pm 16.2$, respectively, which was found to have a statistically significant difference $(p<0.001)$. The participants performed better in the control phase with a mean percentage accuracy of $93.4 \pm 2.1$ (in \%) as compared to accuracy of response performance of $88 \pm 1.3$ (in \%) after exercise cognitive phase but with slow reaction time. 
Fig. 1. P300 latencies differences in study participants in control and exercise phase.

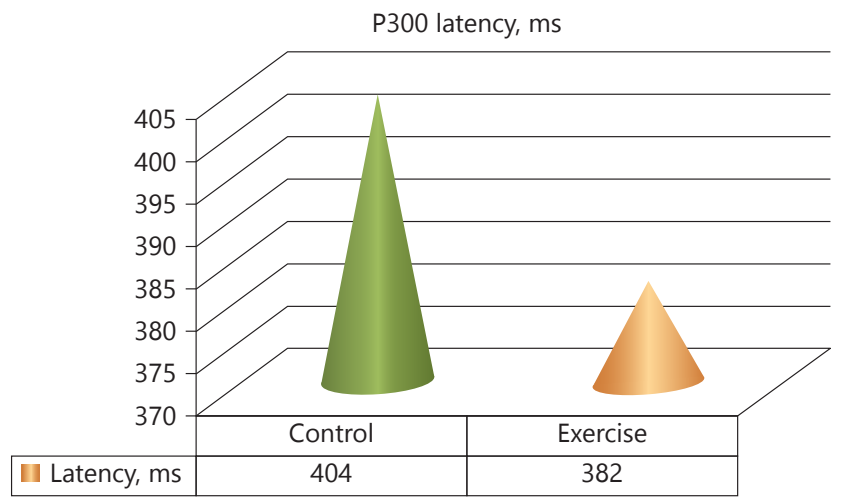

Fig. 2. ERP-P300 amplitude differences in study participants in control and exercise phase.

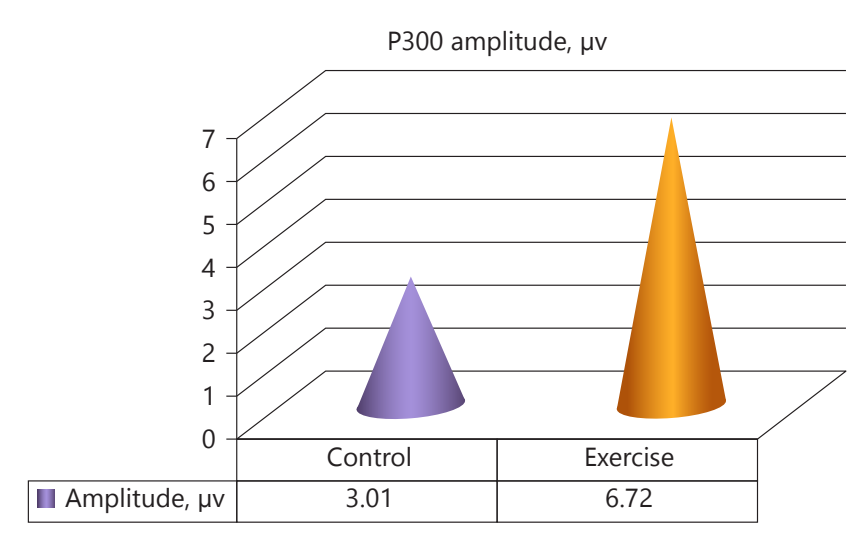

Table 1. Exhaustion characteristics of the participants under control and exercise phase prior to (Pre) and after (Post) performance of the cognitive test

\begin{tabular}{llllll}
\hline MFI-20 & \multicolumn{2}{l}{ Control phase, mean \pm SD } & & \multicolumn{2}{l}{ Exercise phase, mean \pm SD } \\
\cline { 2 - 5 } & pre & post & pre & post \\
\hline Mental exhaustion & $4.3 \pm 1.5^{*}$ & $11.3 \pm 1.8^{\dagger}$ & & $7.4 \pm 2.2^{*}$ & $14.8 \pm 2.9^{\dagger}$ \\
Physical exhaustion & $5.7 \pm 2.1^{*}$ & $7.1 \pm 2.6^{*}$ & & $12.3 \pm 2.7^{*}$ & $15.4 \pm 2.3^{*}$ \\
\hline
\end{tabular}

* Denotes a statistical significance at $p<0.01$ between the means of the MFI measures for precontrol and preexercise cognitive tasks.

$\dagger$ Denotes a statistical significance at $p<0.05$ between the means of the MFI measures for postcontrol and postexercise cognitive tasks.

MFI, multidimensional fatigue inventory. 
Table 2. The cognitive performance of the participants in terms of reaction time and percentage accuracy

\begin{tabular}{llll}
\hline Cognitive tasks & $\begin{array}{c}\text { Control trial, } \\
\text { mean } \pm \text { SD }\end{array}$ & $\begin{array}{l}\text { Exercise trial, } \\
\text { mean } \pm \text { SD }\end{array}$ & $p$ value \\
\hline Reaction time & $470 \pm 19.1$ & $405.2 \pm 16.2$ & $<0.001$ \\
Accuracy, \% & $93.4 \pm 2.1$ & $88 \pm 1.3$ & 0.236 \\
\hline
\end{tabular}

\section{Discussion}

The present study showed the mental and physical exhaustion of the participants were significantly more in the exercise phase cognitive tasking. Interestingly, the participants subjected to the exercise involved cognitive tasking had an overall lower mean reaction time as compared in the control phase. Previous research assessing the relationship between cognitive tasking and reaction time has observed a similar findings after a medium or long duration physical task performance $[8,9,13]$.

The current study showed that the participants were more accurate in their responses during the control phase than in the exercise phase cognitive test tasking. Lorist et al. [24] showed that increase in fatigue leads to decreased percentage accuracy in response which is in agreement with our study [23]. The P300 component mean amplitude was comparatively greater, and its corresponding latency was smaller after the exercise trial than in the control phase, which indicates the participants reached a faster and higher attention state in the exercise trial than in the control phase. The observations were in agreement with that of similar study conducted elsewhere [31]. The P300 amplitude increased following an exercise; however, other researchers have found that P300 amplitude increase is also influenced by mental fatigue [32].

Exercise improves the blood supply to the brain, leading to improved performance in various cognitive tests. Physical activity is related to changes in the brain through overall cardiovascular conditioning, and it enhances cerebral blood flow and oxygen supply to neurons [33]. Chronic exercise is found to be associated with an improvement in spatial memory and positive cholinergic effects [17]. In any case, it is apparent that even a single bout of moderate intensity exercise can bring about an improvement in cognition, and we believe that this is very important, as aerobic exercise could have a dual benefit, both for physical and mental health [18-21]. It is an already established fact that chronic exercise definitely ben- efits cognition. However, it is an interesting incentive for the subject to note that there is an improvement in cognition with even a single bout of exercise.

\section{Conclusion}

Within the limits of the present study, participants experienced more physical and mental exhaustion during the exercise that involved cognitive tasks. Lower P300 latencies reflect faster reaction time, but their response accuracies were poorer resulting in poorer cognitive performances. An apparent decrease in attention based on decreased percentage accuracy of response was evident. Participants subjected to control trial performed better in terms of higher percentage accuracy but with slow reaction times than after subjecting for exercise-induced exhaustion. This implies that exhaustion, performance, and attention are interdependent, and one should work harder to sustain performance levels that would intensify the development of fatigue.

\section{Disclosure Statement}

Authors have no competing financial interests.

\section{Funding Sources}

The authors have no funding to declare.

\section{Author Contributions}

A.B.H.I. and N.A.P.: conceptualized the paper, S.K.R. and R.S.P.: drafted the paper, and R.K.K., A.B.H.I., and S.A.: edited it.
References
1 Ishii A, Tanaka M, Watanabe Y: Neural mechanisms of mental fatigue. Rev Neurosci 2014;25:469-479.
2 Bartlett FC: Fatigue following highly skilled work, Proc. R Soc 1943;131:247-257.
3 Watanabe Y: Preface and mini-review: fatigue science for human health in: Watanabe $\mathrm{Y}$, Evengård B, Natelson $\mathrm{BH}$, et al (eds): Fatigue Science for Human Health (ed 1). New York, Springer, 2008, pp 5-11.
4 Wessely S: The epidemiology of chronic fatigue syndrome. Epidemiol Rev 1995;17:139-151
5 Chaudhuri A, Behan PO: Fatigue in neuro- logical disorders. Lancet 2004; 363:978-988.
6 Brown ID: Driver fatigue. Hum Factors 1994; 36:298-314.

Physical Exhaustion Induced Variations in ERP and Cognitive Tasking 
7 Dosher BA, Lu ZL: Mechanisms of perceptual attention in precuing of location. Vision Res 2000;40:1269-1292.

8 Murray SO, Wojciulik E: Attention increases neural selectivity in the human lateral occipital complex. Nat Neurosci 2004;7:70-74.

9 Posner MI, Snyder CR, Davidson BJ: Attention and the detection of signals. J Exp Psychol 1980;109:160-174.

10 Sellers EW, Donchin E: A P300-based braincomputer interface: initial tests by ALS patients. Clin Neurophysiol 2006;117:538-548.

11 Colcombe S, Kramer AF: Fitness effects on the cognitive function of older adults: a metaanalytic study. Psychol Sci 2003;14:125-130.

12 Tomporowski PD: Effects of acute bouts of exercise on cognition. Acta Psychol (Amst) 2003;112:297-324.

13 Persson J, Welsh KM, Jonides J, Reuter-Lorenz PA: Cognitive fatigue of executive processes: interaction between interference resolution tasks. Neuropsychologia 2007;45: 1571-1579.

14 Boksem MA, Meijman TF, Lorist MM: Mental fatigue, motivation and action monitoring. Biol Psychol 2006;72:123-132.

15 van der Linden D, Frese M, Meijman TF: Mental fatigue and the control of cognitive processes: effects on perseveration and planning. Acta Psychologica (Amst) 2003;113:45-65.

16 Hockey GR: Compensatory control in the regulation of human performance under stress and high workload; a cognitive-energetical framework. Biol Psychol 1997;45:7393.

17 Vogt T, Herpers R, Scherfgen D, Strüder HK, Schneider S: Neuroelectric adaptations to cognitive processing in virtual environments: an exercise-related approach. Exp Brain Res 2015;233:1321-1329.

18 Kumar N, Singh M, Sood S, Beena, Sakshi, Roy PS, et al: Effect of acute moderate exercise on cognitive P300 in persons having sedentary lifestyles. Int J Appl Basic Med Res 2012; 2:67-69.

19 Kumar N, Sood S, Singh M, Beena, Sakshi: Effect of acute moderate exercise on cognitive event-related potentials n100, p200, n200, and interpeak latencies. Indian J Psychol Med 2010;32:131-135

20 Nanda B, Balde J, Manjunatha S: The acute effects of a single bout of moderate-intensity aerobic exercise on cognitive functions in healthy adult males. J Clin Diagn Res 2013;7: 1883-1885.

21 Pontifex MB, Parks AC, Henning DA, Kamijo K: Single bouts of exercise selectively sustain attentional processes. Psychophysiology 2015;52:618-625

22 Smets EM, Garssen B, Bonke B, De Haes JC: The multidimensional fatigue inventory (MFI) psychometric qualities of an instrument to assess fatigue. J Psychol Res 1995;39: 315-325.

23 Lorist MM, Klein M, Nieuwenhuis S, De Jong R, Mulder G, Meijman TF: Mental fatigue and task control: planning and preparation. Psychophysiology 2000;37:614-625.

24 Polich J: Updating P300: an integrative theory of P3a and P3b. Clin Neurophysiol 2007;118: 2128-2148.

25 Furdea A, Halder S, Krusienski DJ, Bross D, Nijboer F, Birbaumer N, et al: An auditory oddball (P300) spelling system for brain- computer interfaces. Psychophysiology 2009; 46:617-625.

26 Henik A: Paying attention to the Stroop effect? J Int Neuropsychol Soc 1996;2:467470.

27 Folstein MF, Folstein SE, McHugh PR: "Minimental state". A practical method for grading the cognitive state of patients for the clinician. J Psychiatr Res 1975;12:189-198.

28 Tombaugh TN, McIntyre NJ: The mini-mental state examination: a comprehensive review. J Am Geriatr Soc 1992;40:922-935.

29 Sanchez-Cubillo I, Perianez JA, AdroverRoig D, Rodriguez-Sanchez JM, Rios-Lago M, Tirapu J, Barcelo F: Construct validity of the trail making test: role of task-switching, working memory, inhibition/interference control, and visuomotor abilities. J Int Neuropsychol Soc 2009; 15:438-450.

30 Lord SW, Clayton RH, Hall MC, Gray JC, Murray A, McComb JM, Kenny RA: Reproducibility of three different methods of measuring baroreflex sensitivity in normal subjects. Clin Sci (Lond). 1998;95:575-581.

31 Nash AJ, Williams CS: Effects of preparatory set and task demands on auditory event-related potentials. Biol Psychol 1982; $15: 15-31$.

32 Nakamura $Y$, Nishimoto K, Akamatu M, Takahashi M, Maruyama A: The effect of jogging on P300 event related potentials. Electromyogr Clin Neurophysiol 1999;39:71-74.

33 Koyama H, Fukuda S, Shoji T, Inaba M, Tsujimoto Y, Tabata T, et al: Fatigue is a predictor for cardiovascular outcomes in patients undergoing hemodialysis. Clin J Am Soc Nephrol 2010;5:659-666. 